\title{
COMMENT
}

\section{Preventing long-term respiratory morbidity in preterm neonates: is there a path forward?}

\author{
Jonathan M. Davis ${ }^{1}$ and DeWayne M. Pursley ${ }^{2}$ on behalf of the Pediatric Policy Council \\ Pediatric Research (2020) 87:9-10; https://doi.org/10.1038/s41390-019-0641-z
}

Bronchopulmonary dysplasia (BPD) is a form of chronic lung disease that develops primarily in preterm neonates. The pathogenesis of BPD is multifactorial with contributors such as positive-pressure ventilation, oxygen toxicity, prematurity, genetic predisposition, infection, inflammation, and nutritional deficiencies appearing to play critical roles. ${ }^{1}$ The early use of less invasive treatment modalities (e.g., nasal continuous positive airway pressure (nCPAP)) and enhanced nutritional approaches (e.g., total parenteral nutrition (TPN), trophic enteral feedings) have resulted in the increased survival of extremely preterm neonates. Despite this, $40-60 \%$ of these neonates will develop BPD with approximately 15,000 new cases reported in the United States each year. BPD is associated with significant morbidity (respiratory, neurodevelopmental), mortality, and poor growth. ${ }^{1}$ The treatment of preterm neonates with BPD is often directed toward improving the pathophysiologic abnormalities after they occur. Additional strategies to prevent BPD and its associated morbidities are urgently needed.

BPD has traditionally been defined as the need for supplemental oxygen at 36 weeks postmenstrual age. ${ }^{2}$ However, this definition has been the subject of intense debate as investigators attempt to link the development of BPD with more meaningful short- and longer-term clinically relevant outcomes, such as: (1) a requirement for home oxygen, (2) development of asthma, (3) repeated respiratory infections, (4) use of respiratory medications, (5) emergency room visits, and (6) hospital re-admissions. ${ }^{3}$ Because many of these outcomes commonly occur in preterm neonates without BPD, the contribution of BPD to the development of long-term respiratory dysfunction is unclear. These morbidities can even be seen in preterm neonates who never required positive-pressure ventilation. ${ }^{4}$ Despite this, BPD remains an extremely important sequela of neonates who require intensive care and is the only major complication of prematurity that continues to increase in frequency. ${ }^{5}$

In this issue of Pediatric Research, Panagiotounakou and colleagues evaluated respiratory function by spirometry and examined the frequency of lower respiratory tract infections, number of re-hospitalizations, and growth parameters in 108 former very preterm neonates (birth weight $<1500 \mathrm{~g}$ and gestational age $<32$ weeks) from a single neonatal intensive care unit (NICU) in Greece and compared them to 70 term controls at 8 years of age. ${ }^{6}$ When the preterm and term groups were compared, there were no significant differences found in forced expiratory volume in $1 \mathrm{~s}\left(\mathrm{FEV}_{1}\right)$ and forced vital capacity measured by spirometry nor respiratory tract infections and rehospitalizations up to 8 years of age. Also important was that no differences could be detected in any respiratory outcome in former preterm neonates who did and did not have a diagnosis of BPD, even though those with BPD had a significantly lower birth weight and gestational age. The authors suggested that their clinical practice of using nCPAP (with permissive hypercapnia) and enhanced delivery of protein (via TPN and early enteral feeds) was primarily responsible for the improved lung function at 8 years of age. These are care practices that have been adopted in many NICUs worldwide. While it is difficult to separate these two approaches from other potentially confounding clinical variables in the NICU as well as various post-discharge factors such as exposure to viral infections, cigarette smoke, and other environmental toxins, these data are provocative and worthy of more in-depth discussion.

It has been over 30 years since Dr. Mary Ellen Avery observed that NICUs using more nCPAP have better respiratory outcomes than those using conventional mechanical ventilation. ${ }^{7}$ This has been followed by clinical trials such as SUPPORT which demonstrated that neonates treated with early nCPAP had better shortand longer-term respiratory outcomes than neonates treated with intubation and exogenous surfactant. ${ }^{8,9}$ This resulted in a major shift in treatment approach with a focus on less invasive ventilatory strategies. However, a recent study by Doyle et al. reported contrasting results. They compared short- (incidence of $\mathrm{BPD})$ and long-term (measurements of airflow) respiratory outcomes at 8 years of age in preterm neonates born in three time periods: $1991-92,1997$, and $2005 .{ }^{10}$ Despite a substantial increase in the use of nCPAP in the later time period, respiratory outcomes had not improved compared to periods when more intubation and surfactant was used.

Preterm neonates have increased nutritional requirements because of increased metabolic needs and rapid growth requirements. If these increased energy and protein needs are not met, a catabolic state will develop, which likely contributes to the pathogenesis of BPD. Inadequate nutrition interferes with normal growth and maturation of the lung and may potentiate the deleterious effects of oxygen and mechanical ventilation. Osborn et al. performed a Cochrane Review to determine whether higher versus lower intake of amino acids administered through TPN is associated with improved growth and reduced incidence of complications, such as BPD. ${ }^{11}$ Data demonstrated varying effects on growth parameters and no significant impact on any

\footnotetext{
${ }^{1}$ Department of Pediatrics, The Floating Hospital for Children at Tufts Medical Center, The Tufts Clinical and Translational Research Institute, Tufts University School of Medicine, Boston, MA, USA and ${ }^{2}$ Department of Neonatology, Beth Israel Deaconess Medical Center, Department of Pediatrics, Harvard Medical School, Boston, MA, USA Correspondence: Jonathan M. Davis (jdavis@tuftsmedicalcenter.org)

Members of the Pediatric Policy Council are listed at the end of the paper.

These authors contributed equally: Jonathan M. Davis, DeWayne M. Pursley
}

Received: 6 October 2019 Accepted: 10 October 2019

Published online: 7 November 2019 
anthropometric measurements at any time point. Higher amino acid intake did not reduce the number of days to full enteral feeds or the incidence and severity of BPD. In addition, Tottman et al. conducted a retrospective cohort study of preterm neonates before and after the introduction of a protocol that administered higher concentrations of protein in the first week of life. ${ }^{12}$ While higher protein intake did not significantly impact growth or neurodevelopmental impairment at 7 years of age, it was concerning that an extra $\mathrm{g} / \mathrm{kg} / \mathrm{day}$ of protein intake was associated with a $27 \%$ increase in the odds of developing cerebral palsy. The study by Panagiotounakou et al. did not report any neurodevelopmental outcomes, findings that are important given the link between abnormal pulmonary and neurodevelopmental outcome in neonates with severe BPD. ${ }^{13}$

Although there have been many clinical trials of new drugs and devices designed to prevent BPD and associated long-term respiratory morbidity, there have been no agents approved by the Food and Drug Administration (FDA) or other global regulatory agencies in the past 20 years for this indication in preterm neonates. Clinical trials of inhaled nitric oxide, recombinant human superoxide dismutase, recombinant human club cell protein, corticosteroids, and novel ventilatory techniques that failed to demonstrate a significant improvement in BPD represent an enormous commitment by families who participated in these research studies as well as the considerable resources needed to support them. The International Neonatal Consortium (INC) was developed as a priority of the FDA and other global regulatory agencies that recognized the need to develop new therapeutic strategies to improve the outcomes of preterm neonates. ${ }^{14}$ INC, a public-private partnership composed of approximately 300 key stakeholders from 25 countries includes the academic community, industry, parent and nursing groups, and many others. INC is focused on developing clinically meaningful outcome measures for clinical trials of BPD and other important complications of neonatal intensive care. With respect to INC's approach to BPD, Steinhorn et al. published a "white paper" outlining a path forward that proposed: (1) better definitions of BPD, (2) more appropriate endpoints for clinical trials, (3) a closer examination of perinatal risk factors for lethal disease, (4) research priorities, and (5) enriching the population of the highest-risk preterm neonates in order to prevent and/or ameliorate the development of BPD and associated long-term pulmonary dysfunction. ${ }^{3}$ Although this thoughtful and comprehensive approach to preventing BPD is critical, a more thorough and detailed analysis of the data from studies such as that reported by Panagiotounakou et al. may be a good place to start. Even though these data come from a single center; may not be generalizable; and likely involve multiple prenatal, postnatal, and environmental factors, it is important to better understand why their outcomes are so positive and unique. There are marked differences in various outcomes across the globe that should be examined in order to truly define "best practices." ${ }^{15}$ This is why organizations such as INC have recommended that neonatal clinical trials need to be conducted in multiple countries simultaneously in order for results to be truly generalizable. Sometimes the simplest approaches turn out to be the best.

\section{MEMBERS OF THE PEDIATRIC POLICY COUNCIL}

Scott C. Denne, MD, Chair, Pediatric Policy Council; Shale L. Wong, MD, MSPH, Representative to the PPC from the Academic Pediatric Association; Jean L. Raphael, MD, MPH, Representative to the PPC from the Academic Pediatric Association; Jonathan Davis, MD, Representative to the PPC from the American Pediatric Society; DeWayne Pursley, MD, MPH, Representative to the PPC from the American Pediatric Society; Tina Cheng, MD, MPH, Representative to the PPC from the Association of Medical School Pediatric Department Chairs; Michael Artman, MD, Representative to the PPC from the Association of Medical School Pediatric Department Chairs; Shetal Shah, MD, Representative to the PPC from the Society for Pediatric Research; Joyce Javier, MD, MPH, MS, Representative to the PPC from the Society for Pediatric Research.

\section{ADDITIONAL INFORMATION}

Competing interests: The authors declare no competing interests.

Publisher's note Springer Nature remains neutral with regard to jurisdictional claims in published maps and institutional affiliations.

\section{REFERENCES}

1. Smith, A. M. \& Davis, J. M. in Manual of Neonatal Respiratory Care 2017 4th edn (eds Donn, S. M. \& Sinha, S. K.) 643-649 (Springer International Publishing AG, Sham, 2017).

2. Jobe, A. H. \& Bancalari, E. Bronchopulmonary dysplasia. Am. J. Respir. Crit. Care Med. 163, 1723-1729 (2001).

3. Steinhorn, R. et al. Bronchopulmonary dysplasia and chronic pulmonary insufficiency of prematurity: developing optimal endpoints for drug development. J. Pediatr. 191, 15-21 (2017).

4. Corwin, B. K., Trembath, A. N. \& Hibbs, A. M. Bronchopulmonary dysplasia appropriateness as a surrogate marker for long-term pulmonary outcomes: a systematic review. J. Neonatal Perinat. Med. 11, 121-130 (2018).

5. Stoll, B. J. et al. Trends in care practices, morbidity, and mortality of extremely preterm neonates, 1993-2012. JAMA 314, 1039 (2015).

6. Panagiotounakou, P. et al. Very preterm neonates receiving 'aggressive' nutrition and early nCPAP had similar long term respiratory outcomes as term neonates. Pediatr. Res. https://doi.org/10.1038/s41390-019-0514-5 (2019).

7. Avery, M. E. et al. Is chronic lung disease in low birth weight infants preventable? A survey of eight centers. Pediatrics 79, 26-30 (1987).

8. Finer, N. N. et al. Early CPAP versus surfactant in extremely preterm infants. N. Engl. J. Med. 362, 1970-1979 (2010).

9. Stevens, T. P. et al. Respiratory outcomes of the surfactant positive pressure and oximetry randomized trial (SUPPORT). J. Pediatr. 165, 240-249 (2014).

10. Doyle, L. W. et al. Ventilation in extremely preterm infants and respiratory function at 8 years. N. Engl. J. Med. 377, 329-337 (2017).

11. Osborn, D. A., Schindler, T., Jones, L. J., Sinn, J. K. \& Bolisetty, S. Higher versus lower amino acid intake in parenteral nutrition for newborn infants. Cochrane Database Syst. Rev. 3, CD005949 (2018).

12. Tottman, A. C. et al. Relationships between early neonatal nutrition and neurodevelopment at school age in children born very preterm. J. Pediatr. Gastroenterol. Nutr. https://doi.org/10.1097/MPG.0000000000002471 (2019).

13. Nakanishi, H., Uchiyama, A. \& Kusuda, S. Impact of pulmonary hypertension on neurodevelopmental outcome in preterm infants with bronchopulmonary dysplasia: a cohort study. J. Perinatol. 36, 890-896 (2016).

14. Turner, M. A. et al. The International Neonatal Consortium: collaborating to advance regulatory science for neonates. Pediatr. Res. 80, 462-464 (2016).

15. Draper, E. S. et al. Variability in very preterm stillbirth and in-hospital mortality across Europe. Pediatrics 139, e20161990 (2017). 